\title{
Performance of CMOS sensors for a digital electromagnetic calorimeter
}

\author{
Paul Dauncey* \\ Imperial College London \\ E-mail: P.Dauncey@imperial.ac.uk
}

\begin{abstract}
We have developed monolithic silicon pixel sensors as study devices for a digital electromagnetic calorimetry application at future collider detectors, such as a linear collider. The motivation for a digital ECAL and the sensor requirements which arise from this are discussed.

We present results from the "TPAC" CMOS sensors produced using the $0.18 \mu \mathrm{m}$ INMAPS process. The sensors have $50 \mu \mathrm{m}$ pixel size. The technology is also applicable to tracking and vertexing applications where highly granular pixels with integrated in-pixel readout is needed.

Several varieties of the TPAC sensors were fabricated with and without some of the various processing innovations available in INMAPS, specifically deep P-wells and high-resistivity epitaxial silicon. The performance of these sensor variants has been measured both in the laboratory and at beam tests. Comparisons of these sensors with each other are presented, showing that the INMAPS innovations result in significant improvements in the sensor performance.
\end{abstract}

35th International Conference of High Energy Physics

July 22-28, 2010

Paris, France

* On behalf of the SPiDeR Collaboration; http://www. spider.ac.uk 


\section{Motivation for digital electromagnetic calorimetry}

The planned $e^{+} e^{-}$international linear collider (ILC) [1] will have an energy of $500 \mathrm{GeV}$ to $1 \mathrm{TeV}$, with a physics programme complementary to LHC. Many ILC physics processes are characterised by multi-jet final states so the reconstruction of the invariant mass of two or more jets is critical. Jet energy resolution is a major driver for ILC detector designs [2, 3, 4]. "Particle flow" techniques will provide the required performance but require highly granular calorimeters.

Usually, a sampling electromagnetic calorimeter measures the energy deposited in the sensitive layers using analogue readout. The concept of a digital electromagnetic calorimeter (DECAL) is to measure the number of particles in the sensitive layers instead, which requires digital readout but with much finer granularity. Counting particles eliminates the resolution contribution from Landau fluctuations of the deposited energy and so the resolution should be closer to the intrinsic stochastic shower variations. High granularity, typically $100 \mu \mathrm{m}$, is necessary to reduce the probability of multiple particles per pixel. This should also improve pattern recognition for particle flow.

Simulation comparing analogue and digital calorimeters indicates a significant resolution improvement in principle, typically reducing the stochastic term in $\sigma_{E} / E$ by $20 \%$ for a given sampling fraction, see fig 1 .
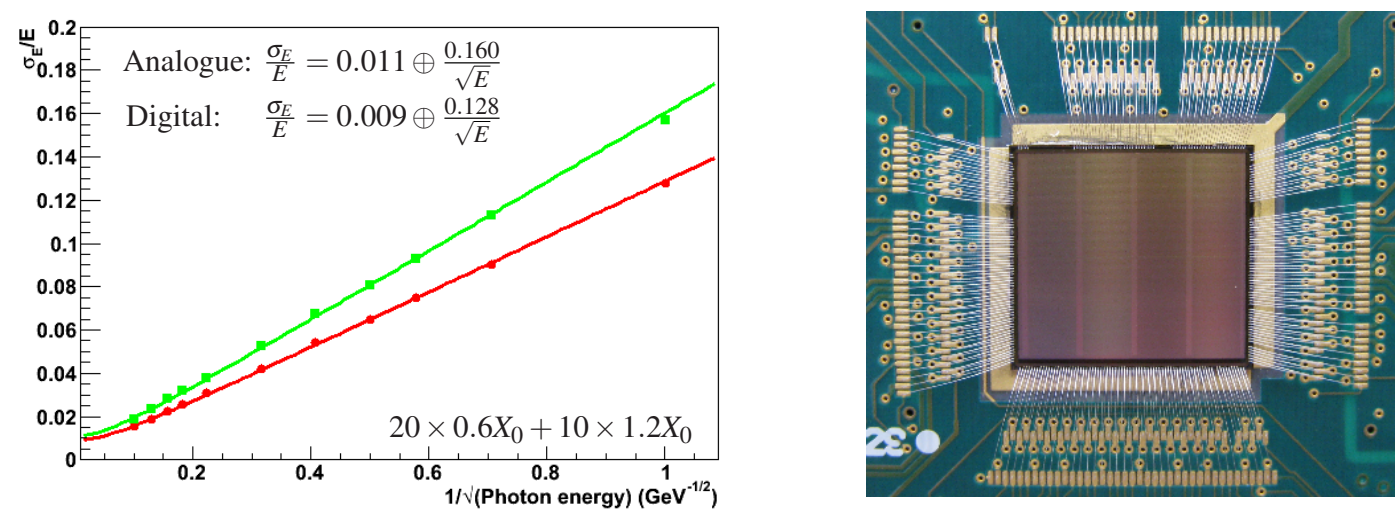

Figure 1: Left: Comparison of $\sigma_{E} / E$ as a function of $1 / \sqrt{E}$ for simulated analogue (green) and digital (red) electromagnetic calorimeters with a typical ILC geometry. Right: Photograph of a TPAC V1.2 sensor.

\section{The TPAC sensor}

The TPAC (Tera-Pixel Active Calorimeter) sensor V1.2 [5], shown in fig. 1, is a study sensor to investigate aspects of digital electromagnetic calorimetry. It was fabricated using the $0.18 \mu \mathrm{m}$ "INMAPS" CMOS process, which has enhanced features beyond standard CMOS. Specifically, INMAPS includes deep P-well to prevent signal absorption in circuit $\mathrm{N}$-wells, hence allowing use of full CMOS (NMOS and PMOS) in-pixel. It also allows the use of high-resistivity silicon in the epitaxial layer for faster signal collection.

The TPAC V1.2 senor has a $50 \mu \mathrm{m}$ pixel pitch on a $1 \mathrm{~cm}^{2}$ sensor, giving a $168 \times 168$ pixel array, with 28k pixels total. A total of four variants of sensors were fabricated, with and without INMAPS enhancements, namely; standard CMOS, INMAPS deep P-well, and INMAPS deep Pwell plus high-resistivity epitaxial layer, with standard $(12 \mu \mathrm{m})$ and thicker $(18 \mu \mathrm{m})$ layer depth. 


\section{CERN and DESY beam tests and results}

All four variants of TPAC V1.2 sensors were tested in beam at CERN (August 2009) and DESY (March 2010), using $120 \mathrm{GeV}$ pion beams at CERN and 1-5 GeV electron beams at DESY. A stack of six sensors was exposed to the beams. Tracks were formed from hits in combinations of sensors and projected to each layer to measure the probability of a pixel hit as a function of distance from the track, as shown in fig. 2. The MIP efficiency was then determined by fitting this distribution, allowing for track resolution.
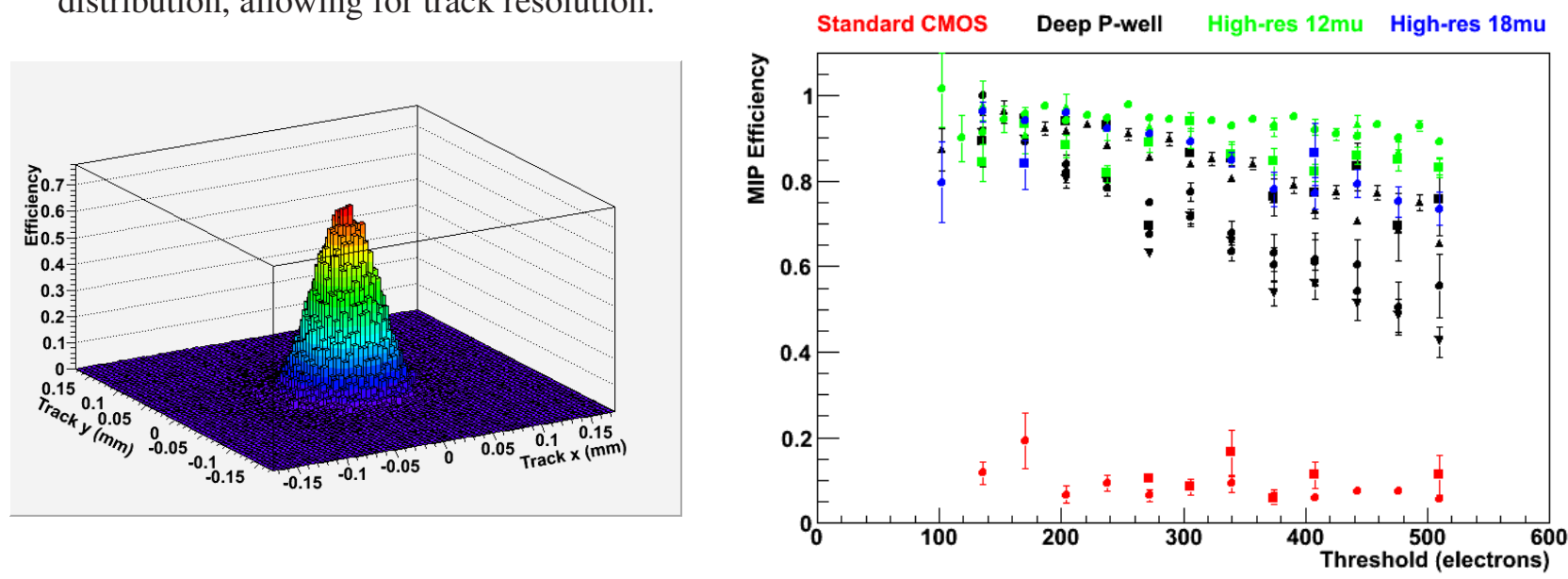

Figure 2: Left: Typical distribution for the probability of a hit as a function of position relative to the track projection. Right: MIP efficiency as a function of threshold, for all four sensor variants.

The efficiency was found as a function of the digital threshold. A MIP leaves an average signal of 1300 electrons in a $12 \mu \mathrm{m}$ epitaxial layer and, due to charge spread, at most half of this is absorbed by any single pixel. The threshold was varied between 100 and 500 electrons, which are typical values needed for a DECAL.

The results of this analysis are shown in fig. 2. The standard CMOS sensors have low efficiency at all thresholds, as expected due to use of in-pixel PMOS transistors. The deep P-well reduces $\mathrm{N}$-well signal absorption and so raises the efficiency very significantly (to nearly $100 \%$ for low thresholds). Adding the high-resistivity epitaxial layer makes further improvements with the resulting efficiency being close to $100 \%$ across most of the threshold range.

\section{Conclusions}

Very significant improvement in efficiency have been demonstrated using INMAPS-enhanced features compared to standard CMOS process. This allows the use of in-pixel PMOS components, enabling sophisticated processing per pixel, and shows a complex DECAL sensor is feasible.

\section{References}

[1] J. Brau, Y. Okada and N. Walker (eds.), International Linear Collider Reference Design Report, (2007), http: / /www. linearcollider.org/rdr.

[2] SiD Detector Concept, http://silicondetector.org/. 
[3] ILD Detector Concept, http://www.ilcild.org/.

[4] CALICE Collaboration, https://twiki.cern.ch/twiki/bin/view/CALICE/WebHome.

[5] J. A. Ballin et al., Monolithic Active Pixel Sensors (MAPS) in a quadruple well technology for nearly 100\% fill factor and full CMOS pixels, Sensors 8 (2008) 5336. 